\title{
Deciphering the fluctuations of high frequency birth rates
}

\author{
Claudiu Herteliu ${ }^{1}$, Peter Richmond ${ }^{2}$ and Bertrand M. Roehner ${ }^{3}$
}

\begin{abstract}
Here the term "high frequency" refers to daily, weekly or monthly birth data. The fluctuations of daily birth numbers show a succession of spikes and dips which, at least at first sight, looks almost as random as white noise. However in recent times several studies were published, including by the present authors, which have given better insight into how birth is affected by exogenous factors. One of them concerns the way adverse conditions (e.g. famines, diseases, earthquakes, heat waves) temporarily affect the conception capacity of populations, thus producing birth rate troughs 9 months after mortality waves. In addition, religious interdicts (e.g. during the Lent period) lead to reduced conceptions. These as well as other effects raise the hope that we will soon be able to "read" and interpret birth rate patterns just as the Egyptologist Jean-Francois Champollion managed to decipher many (though not all) hieroglyphs.
\end{abstract}

Version of 20 February 2018

Provisional. Comments are welcome.

Key-words: birth, fluctuations, holidays, religion, mass mortality, heat waves

1: Department of Statistics and Econometrics, Bucharest University of Economic Studies, Bucharest, Romania. Email: claudiu.herteliu@gmail.com

2: School of Physics, Trinity College Dublin, Ireland. Email: peter_richmond@ymail.com

3: Institute for Theoretical and High Energy Physics (LPTHE), University Pierre and Marie Curie, Sorbonne Université, Centre de la Recherche Scientifique (CNRS). Paris, France.

Email: roehner@lpthe.jussieu.fr 


\section{Introduction}

That the determinants of conceptions and births are still not well understood is shown fairly clearly by the fact that measures taken to boost birth rates by governments of countries experiencing shrinking populations (e.g. Japan or Singapore) proved largely ineffective.

The present paper is not focused on such long-term changes but rather on shortand medium-term changes. Nevertheless, some of the effects studied here may also have long-term implications. As an illustration one can mention the effect on births of religious interdicts. So numerous were such interdicts in Middle Age western Europe that only about 70 days were left annually for permitted sexual relations. Had they been strictly followed, such rules would have drastically restricted fertility.

Fig. 1 represents daily birth numbers in France in 1969. This fast succession of upward and downward spikes resembles a graphical representation of white noise except that instead of being stationary the signal displays also some medium-term ups and downs.

To make sense of such a jerky graph seems a real challenge. Usually, the standard approach is to perform a moving window average which will eliminate high frequency changes. The remaining ups and downs will then be interpreted in terms of specific human activities (e.g. vacation, harvesting in rural societies and so on) which are assumed either to favor or reduce conception rates. Most of the time such interpretations remain fairly qualitative (see for instance Houdaille 1979, 1985) for it is hardly possible to know in a quantitative way the effect vacationing or harvesting has on the sexual behavior of people.

The method that will be used here is different in the sense that we will focus our attention on sharply defined events such as epidemics, earthquakes, hot weather days, national or religious celebrations, in an attempt to identify their consequences in terms of birth rate changes nine months later. In other words, we are not interested in ordinary life activities (such as vacationing or harvesting) but rather in exceptional events. In this way we will be able to build a kind of dictionary listing the connections between specific peaks or troughs and the events which triggered them 9 months earlier. As implied by the parallel with Champollion made in the abstract, this dictionary will not give the clue for all accidents observed in birth series. Unexplained spikes or troughs may point the attention of researchers to new, still unknown, phenomena.

The paper proceeds through the following steps.

(1) First, we identify daily spikes attributable to holidays during which medically induced births are drastically reduced. In this way, all national holidays which occur 


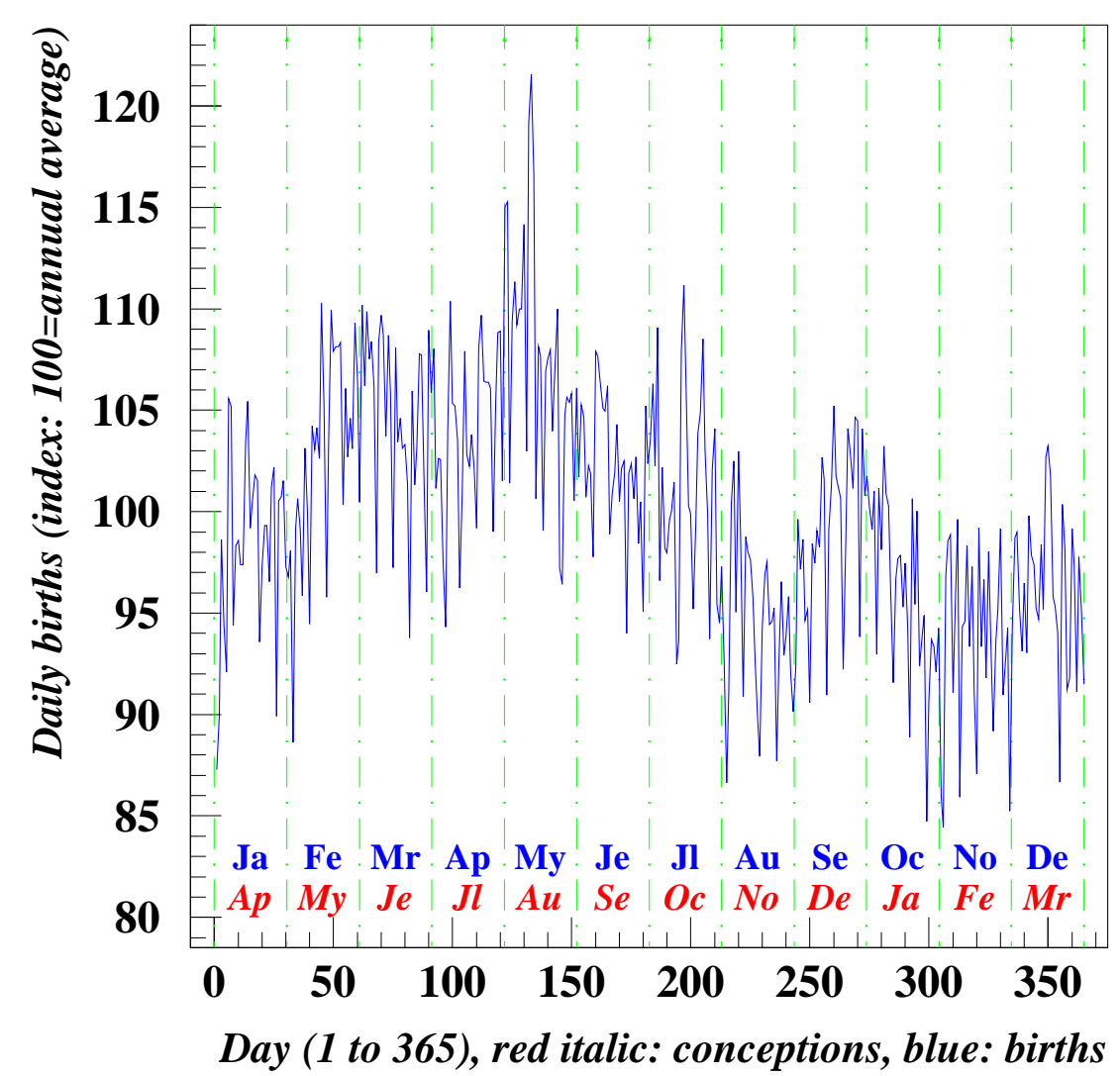

Fig. 1 Daily births in France in 1969. The data were normalized by dividing them by the annual average. Source: Répartition quotidienne des naissances vivantes, France métropolitaine. Institut National de la Statistique et des Études Économiques (INSEE). [Daily distribution of live births. Overseas territories are excluded. National Institute for Statistics and Economic Studies]. Available on Internet under the title: "Les naissances en 2016. Tableaux de séries longues".

on fixed days (such as 4 July in the US or 14 July in France) will become apparent. This is a fairly easy step which only requires an averaging procedure over a sufficiently large number of years.

(2) Secondly, we focus on adverse conditions which create troughs nine months later. This part relies on the results given in a recent paper (Richmond et al. 2018a).

(3) The two previous parts give interpretations of dips and narrow troughs but not of wide troughs nor of peaks. Here we show that broad troughs can result from religious interdicts and that birth peaks may be brought about by religious celebrations.

\section{"Days off" effect}

\section{Identification of the cause of the downward spikes}

Fig. 2a shows the curve that one obtains by summing up 35 annual curves (restricted to non-leap years) such as the one represented in Fig. 1. Not surprisingly, the dis- 
persion is reduced. Whereas the curve of Fig. 1 moves within $100 \pm 10$ the curve of Fig. 2 moves within $100 \pm 5$.

If successive years were independent their average would have a dispersion 6 times (i.e. $\sqrt{35}$ ) smaller than the initial series. The fact that the dispersion is only divided by 2 shows that the successive series are highly correlated; this point was already discussed in Richmond et al. (2018b).

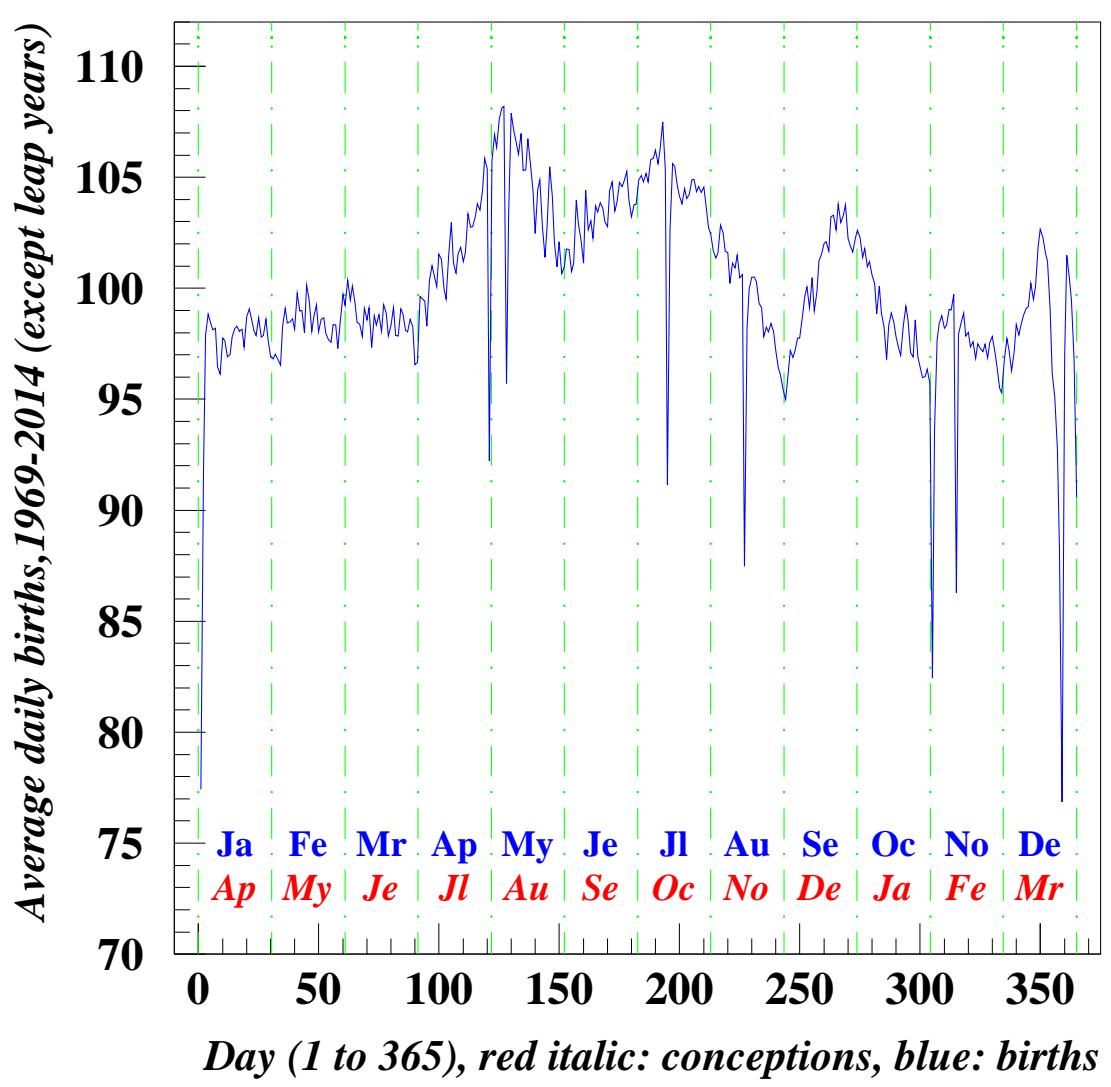

Fig. 2a Average of daily births in France over 35 years, 1 January=1 The data cover 1969-2014 (leap years were excluded). The downward spikes correspond to non-mobile public holidays in France: 1 May is Labor Day, 8 May is end of World War I, 14 Jul is Bastille Day, 1 Nov is in memory of all deceased, 11 Nov is end of World War I, 24-25 Dec is Christmas. Source: Same as for Fig.1.

However, there are downward pointing spikes which reach well beyond this interval. From their positions it is easy to recognize that they correspond to fixed public holidays. The reduction in the number of births on non work days relates to medical induction of labor where drugs are used to induce labor in advance of it beginning naturally. Obviously such interventions will rather be planned on working days. In 2014 some $23 \%$ of American pregnant women experienced labor induction up from a percentage of $10 \%$ in 1990. Cesarean delivery has also increased: from 15\% in 1996 to some $22 \%$ in 2011. Note the figures for induced labour and Cesarian delivery 


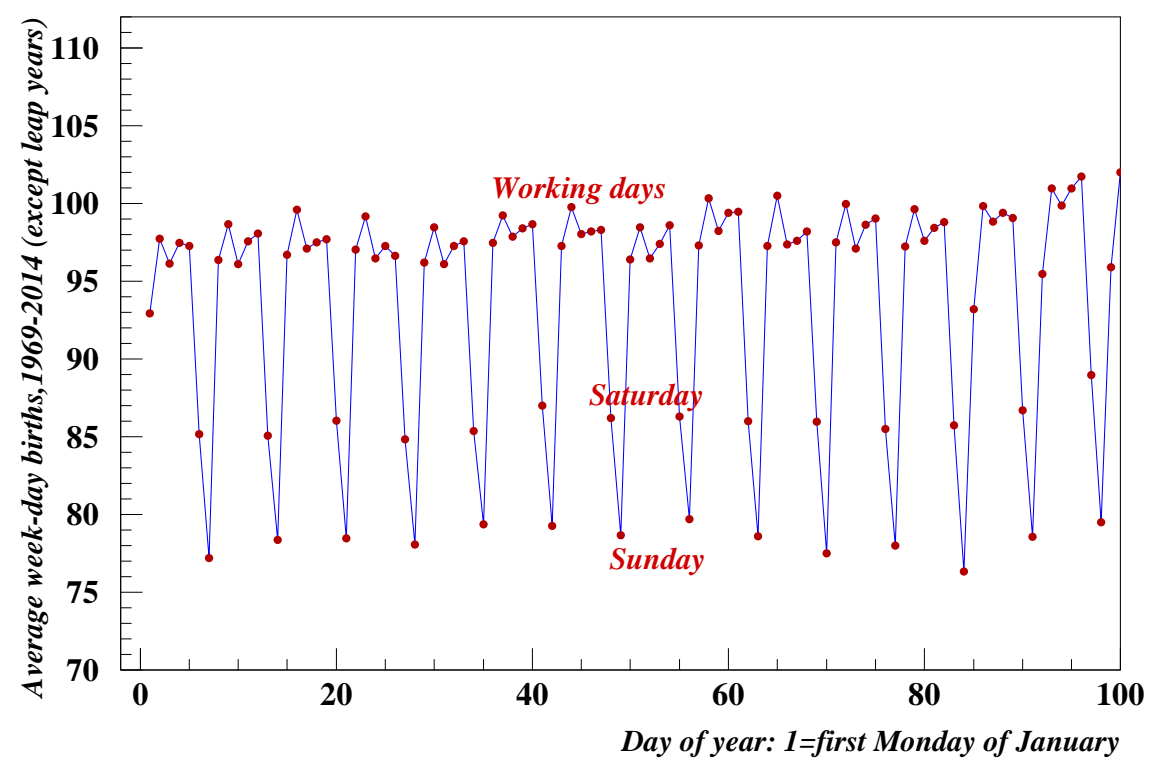

Fig. 2b Average of daily births in France over 35 years starting with first Monday of January. The data cover 1969-2014 (leap years were excluded). The downward spikes correspond to Saturdays and Sundays. It is for the sake of clarity that the series was limited to 100 days (instead of 51 weeks). Source: Same as for Fig.1.

should not be added together since the former may lead to the latter. 1 . The proportion of induced delivery is about the same in France (Enquête nationale périnatale [Perinatal national survey] 2010).

For the period of 35 years following 1969 (excluding leap years) the average reduction in birth numbers for public holidays is about $12 \%$.

\section{Effect of Saturdays and Sundays}

On Fig. 2a one cannot clearly see the effect of mobile public holidays. Examples are Easter or "Labor Day" in the US which is on the first Monday of September. For the same reason, the effect of Saturdays and Sundays does not appear. However, the weekends appear in Fig.2b. Between Fig $2 a$ and $2 b$ there is only a small difference in design but it makes a drastic difference in shape. In Fig.2a, because the annual series start at 1 January which can be any day of the week the weekends are positioned fairly randomly with the result that in successive years the summation mixes weekends and working days. On the contrary in Fig. $2 b$ the annual series start at the first Monday of January with the result that the weekends are at the same locations in successive years; thus, the summation singles them out in a clear way. By direct examination of the data, one finds the following reductions in birth numbers for 1969 and 2016. The increase in the reduction reflects the increase in the proportion of induced and Cesarean deliveries.

\footnotetext{
${ }^{1}$ The sources are: “Quick facts about labor induction”, August 2016; Centers for Disease Control: Recent declines in induction of labor by gestational age, June 2014.
} 
Table 1: Effect of Saturdays and Sundays on birth numbers with respect to working days.

\begin{tabular}{llll}
\hline \hline & Year & Saturday & Sunday \\
\cline { 2 - 4 } & 1969 & $-1.2 \%$ & $-6.8 \%$ \\
2016 & $-15 \%$ & $-19 \%$ \\
\hline
\end{tabular}

Notes: The data are for France. The reduction indicated in the table refers to the difference between Saturday or Sunday and the average of adjacent working days.

Sources: Same as for Fig. 1. The topic of weekend birth rates is also studied and discussed in Lerchl (2005), Lerchl et al.(2008) and Lerchl (2008).

\section{Removal of the "Days off" effect}

In order to estimate the global effect that days off have on birth numbers, we replaced these data by the average of the births observed on adjacent working days. The resulting shape (see Fig. 3) is much less jerky than in Fig. 1.

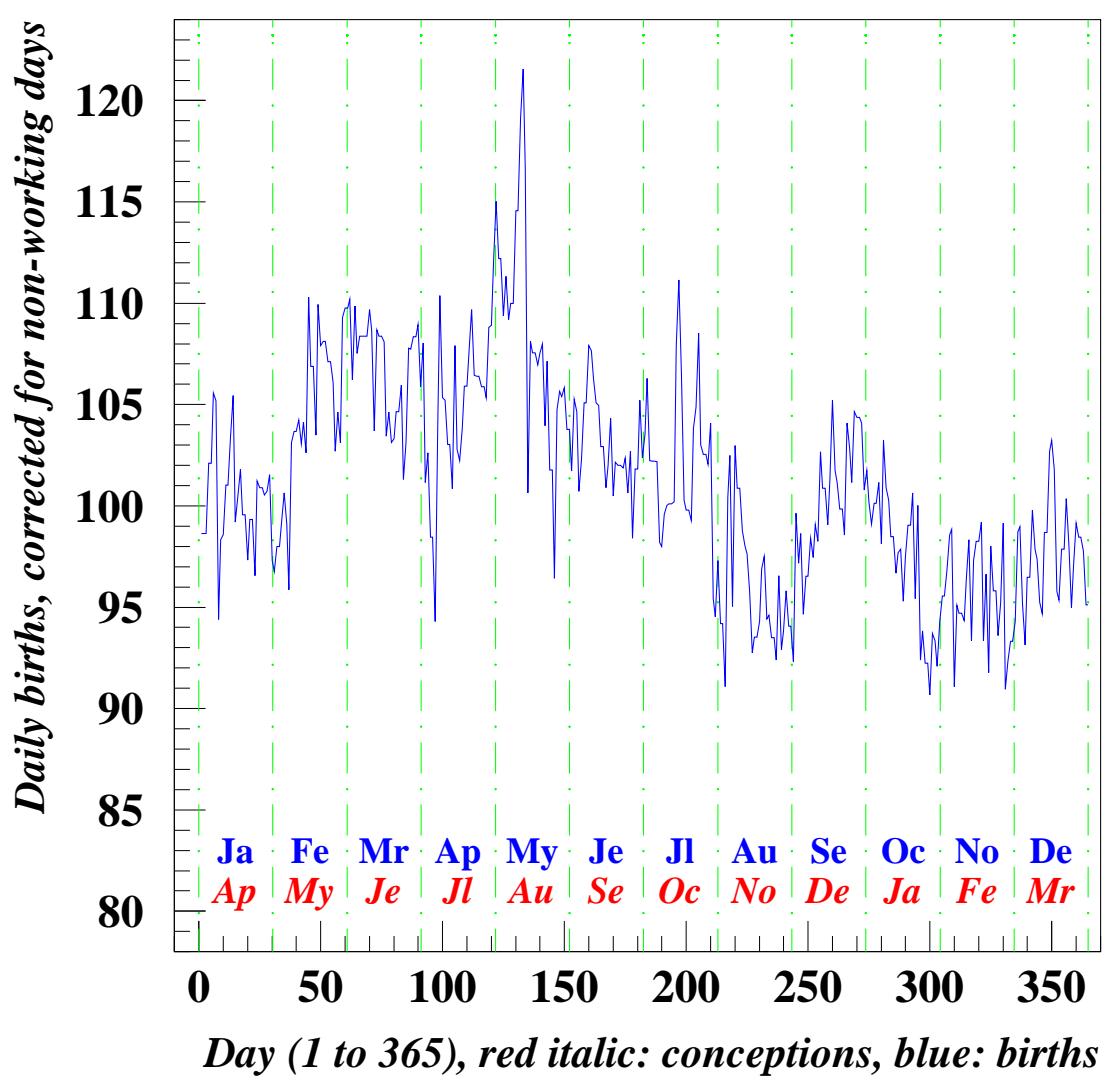

Fig. 3 Daily births in France in 1969 after anomalous non-working day data were smoothed out. Data for days off were replaced by the average of the births on the two adjacent working days. This resulted in a reduction of the standard deviation from 6.12 in Fig. 1 to 5.29 here. Source: Same as for Fig.1.

The removal of the days off was performed in 3 steps which resulted in the following reductions of the standard deviation:

Initial: $\sigma_{1}=6.1$, No public holidays: $\sigma_{2}=6.0$, No Sundays: $\sigma_{3}=5.4$, No Saturdays: $\sigma_{4}=5.3$ 


\section{Effect of adverse living conditions on conceptions}

The removal of the "Days off" effect has a dramatic impact on the shape of the curve of daily births, but one must recognize that it is a fairly obvious effect. Less trivial is the effect resulting from adverse living conditions which most often are revealed through a surge in mortality. This effect is less obvious for two reasons.

- It occurs at time of conception that is to say 9 months before the births.

- From conception to birth there are several successive steps which involve social as well as biological phenomena. It is a comparative analysis of several case studies which revealed that the key-role is played by social rather than biological factors (Richmond et al. 2018a,b).

In this section, this effect will be presented through two cases.

(1) The impact that influenza outbreaks have on conceptions.

(2) The impact that heat waves have on conceptions.

\section{Implication of influenza outbreaks for conceptions}

In Richmond et al. (2018a) the authors used data for influenza outbreaks of exceptional gravity such as in 1889 or 1918 . Here we will consider "ordinary" outbreaks of the kind that occur every year either in December or (most often) in January. Naturally, the effect is less spectacular but this analysis will show that even small outbreaks have a sizable effect on conceptions.

In Richmond et al. (2018a) the focus was on special events which means that we knew in advance the year and month we should consider. Here, on the contrary, there are no special events; thus, how do we know the years and months on which to focus? The following answer relies on the fact that the influenza outbreaks are not the same every year, despite of the fact that their timings are fairly similar.

- For the month, there is little hesitation for one knows that it is January which is the peak month for influenza deaths. It is true that in some years the peak is in December or in February but even in such cases (which are uncommon, less than 20\%) January also has a high death rate (Fig. 7a,b show death rates in the decades 1946-1955, 1996-2005 in France, see also the CDC Wonder database for 1999-2016 in the US).

- As to the year, it should be observed that there are in fact differences from year to year. The strategy here is to select the years which have the highest January death rates. In this respect it is important to observe that there is a high degree of correlation between January death rates either for influenza or for all causes of deaths.

For instance, in the United States in the 18 years from 1999 to 2016 the correlation is as high as 0.82 . This is a remarkable result for influenza and pneumonia (code

\footnotetext{
${ }^{2}$ For the percentage deviations with respect to annual averages the regression reads: $y=a x+b, a=5.9 \pm 2, b= \pm 4$,
} 

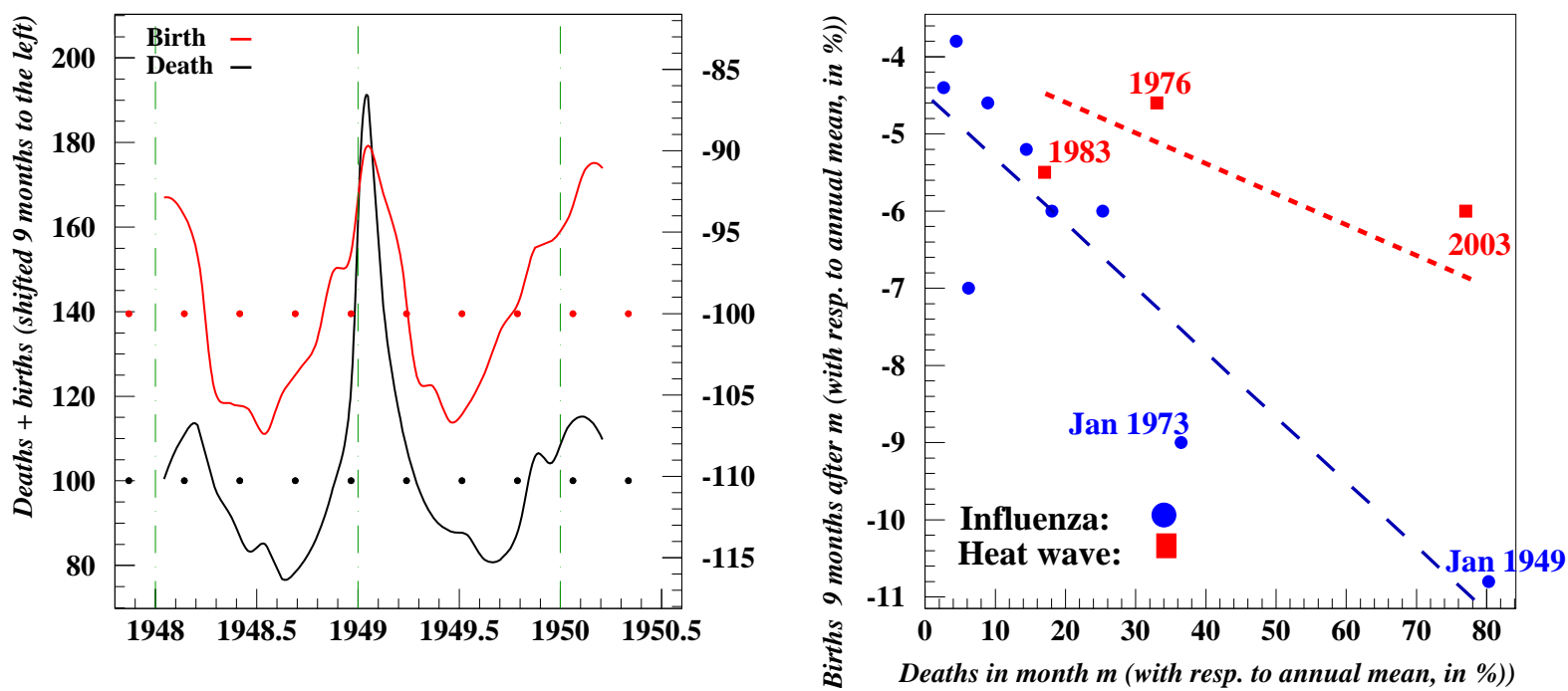

Fig. 4a,b The effect of influenza outbreaks on births in France between 1948 and 1974. Left: Relationship between monthly deaths and monthly births nine months later. Right Relationship between monthly excess deaths in January (with respect to the annual average and reduction in births in October of the same year. The coefficient of correlation is -0.89 and the equation of the regression line is: $y=a x+b, a=-0.084 \pm 0.03, b=$ $-4.5 \pm 0.7$. The three squares in red correspond to heat waves in France (see the text). Sources: Births: Same website as for Fig. 1. Deaths: "Nombre moyen de décès par jour selon le mois depuis 1946 pour la France métropolitaine”, INSEE. [Monthly numbers of deaths divided by the length of each month from 1946 to 2016]. Available on Internet under the title: "Les décès en 2016. Tableaux de séries longues".

J09-18 of the International Classification of Diseases) deaths in fact represent only a small fraction (about 3\%) of the total deaths of January. The fact that despite this small fraction influenza nevertheless controls the January deaths is due to the fact that, apart from influenza changes, the total January deaths are very stable; their coefficient of variation is only about $5 \%$. Thanks to the high correlation, we can select the appropriate years by using either the monthly influenza deaths or, if those data are not available, the monthly totals.

In Fig. 4b we selected the years in the interval 1949-1974 for which the January deaths were higher than the annual average. The plot shows clearly that there is a distinct impact on births for the highest death peaks which are the analogues of the exceptional events studied in Richmond et al. (2018a). For low January death rates, the impact of influenza deaths becomes masked by the background noise. In other words, in such cases the effect may still exist but it is no longer visible.

In the rest of the paper, for the sake of brevity, the effect described here will be called the death-nobirth effect.

\section{How deaths are affected by heat waves}

where $x$ represents excess influenza deaths and $y$ excess deaths for all causes. The data used here are from the CDCWonder database. 
It is a fairly common sense notion that heat waves may affect fragile persons particularly babies and elderly people. Needless to say, one would expect this effect to be more pronounced in countries (like France or Switzerland considered below) where air conditioning is fairly uncommon. Although there have been many papers which document this effect only few give age-specific results. Among the later, there are two fairly recent papers which are particularly commendable in this respect, namely Rey et al. (2007) for France and Vicedo-Cabrera et al. (2016) for Switzerland.

From our present perspective it is particularly important to know what age groups are mostly affected for, if only babies and elderly are affected, there will be no reduction in births nine months later. In Rey et al. (2007) there is a very interesting table which gives all the information that we need.

Table 2: Age distribution of the victims of heat waves and influenza respectively.

\begin{tabular}{lcccc}
\hline \hline & All ages & Age & Age & Age \\
& $0-100$ & $0-35$ & $35-75$ & $75-100$ \\
\hline Deaths during heat waves, av. 1975-2003 (1) & 4,400 & $3.5 \%$ & $24 \%$ & $70 \%$ \\
Deaths in normal conditions, av. 1975-2010 (2) & & $8.0 \%$ & $37 \%$ & $54 \%$ \\
Excess mortality ratio (1)/(2) & & 0.33 & 0.65 & 1.30 \\
& & & \\
Deaths from influenza, av. 1999-2016 (1) & 57,700 & $1.8 \%$ & $23 \%$ & $75 \%$ \\
Deaths in normal conditions, 1999-2016 (2) & & $4.5 \%$ & $39 \%$ & $56 \%$ \\
Excess mortality ratio (1)/(2) & & 0.40 & 0.59 & 1.34 \\
\hline
\end{tabular}

Notes: The heat wave data are for France and are an average of 6 heat waves, namely: 1975, 1976, 1983, 1990, 2001, 2003. The influenza data are for the United States and are an average for 1999-2016. Taking into account that the US population is about 5 times larger than the French, we see that for a same population there are about 2.6 times more influenza deaths than heat wave deaths. Moreover, contrary to influenza outbreaks that occur every year heat waves take place on average only every 5 years. In terms of gender distribution, for heat waves females represented $58 \%$ of the victims, a figure which is not surprising because females are over-represented in the age group over 75 years.

Source: Heat waves: Rey et al. (2007, Table 1). Influenza: CDC-Wonder database.

Table 2 shows that, not surprisingly, young people under 35 are under-represented among heat wave victims while elderly people over 75 are over-represented. However, the same table shows that for influenza the proportions are almost the same. Actually, we are not interested in the persons who die but rather in those who are affected and survive. Thus, the real question is: "Are influenza survivors more affected than heat wave survivors?" By studying the effect on births, we can answer this question at least in its specific meaning of "being affected in terms of conception capacity". This is the point to which we come now.

\section{How conceptions are affected by heat waves}

Whereas there have been many papers about the effect of heat waves on death rates, 
to our best knowledge there was only one author who investigated its effects on birth rates nine months later. In a pioneering study Arnaud Régnier-Loilier analyzed the effect on conceptions and births of three heat waves that occurred in France, namely: 1976, 1983 and 2003. Here the percentages of deaths with respect to annual deaths are of the order of a few percent that is to say about 10 times smaller than for influenza deaths. This makes the analysis more tricky. In order to get rid of the seasonal fluctuations Régnier-Loilier divided the births of the year under consideration by the series of the two adjacent years. This leads to the identification of birth troughs whose width is about 40 days.

Such shocks belong to the same death-nobirth family as those for famine, influenza or earthquakes considered above and in Richmond et al. (2018a). That is why it makes sense to plot the results in the graph of Fig. 4b. We can see that for the same percentage of deaths one gets smaller conception troughs. This provides an answer to the question raised above. We see that (for the same number of fatalities) the heat wave survivors are less affected than the influenza survivors, a rather natural conclusion intuitively.

In conclusion to this subsection about the effect of heat waves, we wish to point out some remaining uncertainties and interrogations.

- The identification of the troughs is somewhat doubtful because, except in 2004, they are not visible on the birth time series: they do not appear on the monthly data and, due to their large fluctuations, even less on the daily data. They become visible only after two operations have been performed. First, each annual birth series $b$ is "renormalized" through division by the average of the two adjacent series, e.g. $b=(1977)$ is replaced by $b^{\prime}=1977 /[(1976+1978) / 2]$. One would expect such a division to reduce the variability (that should be so if the denominator is sufficiently similar to the numerator) but that is not what is observed: actually in the three cases of 1977, 1984, 2004, the coefficient of variation of the initial series is (on average) smaller than the CV of the renormalized series (the ratio is 0.97 ).

The second operation is a broad moving average with a window width of 21 days. These operations lead to series which have more ups and downs than the initial series would have after a similar moving average.

In short, the effect may well exist but due to its small magnitude it is at the limit of being detectable.

- The second interrogation concerns the specific mechanism which brings about the birth troughs. Is it a biological or a behavioral effect? In other words, does the higher temperature affect the conception mechanisms or does it discourage intercourse? The high fertility that one sees in many African countries calls in doubt a biological incidence. The fact that similar birth reductions are observed in the wake 
of earthquakes also weights in favor of the behavioral explanation.

\section{Removal of the death-nobirth effect for daily births}

Now we wish to see how the fluctuations of births shown in Fig.3 are reduced when the death-nobirth effect is removed, that is to say when the dips due to excess-deaths 9 months earlier are compensated for. This compensation process is done by using the linear regression equation given in the the caption of Fig. 4b. The standard deviation is reduced from the previous value of $\sigma_{4}=5.3$ to $\sigma_{5}=4.7$ (a reduction of $11 \%)$. This is a fairly small reduction and Fig. 5 explains why it is not larger.

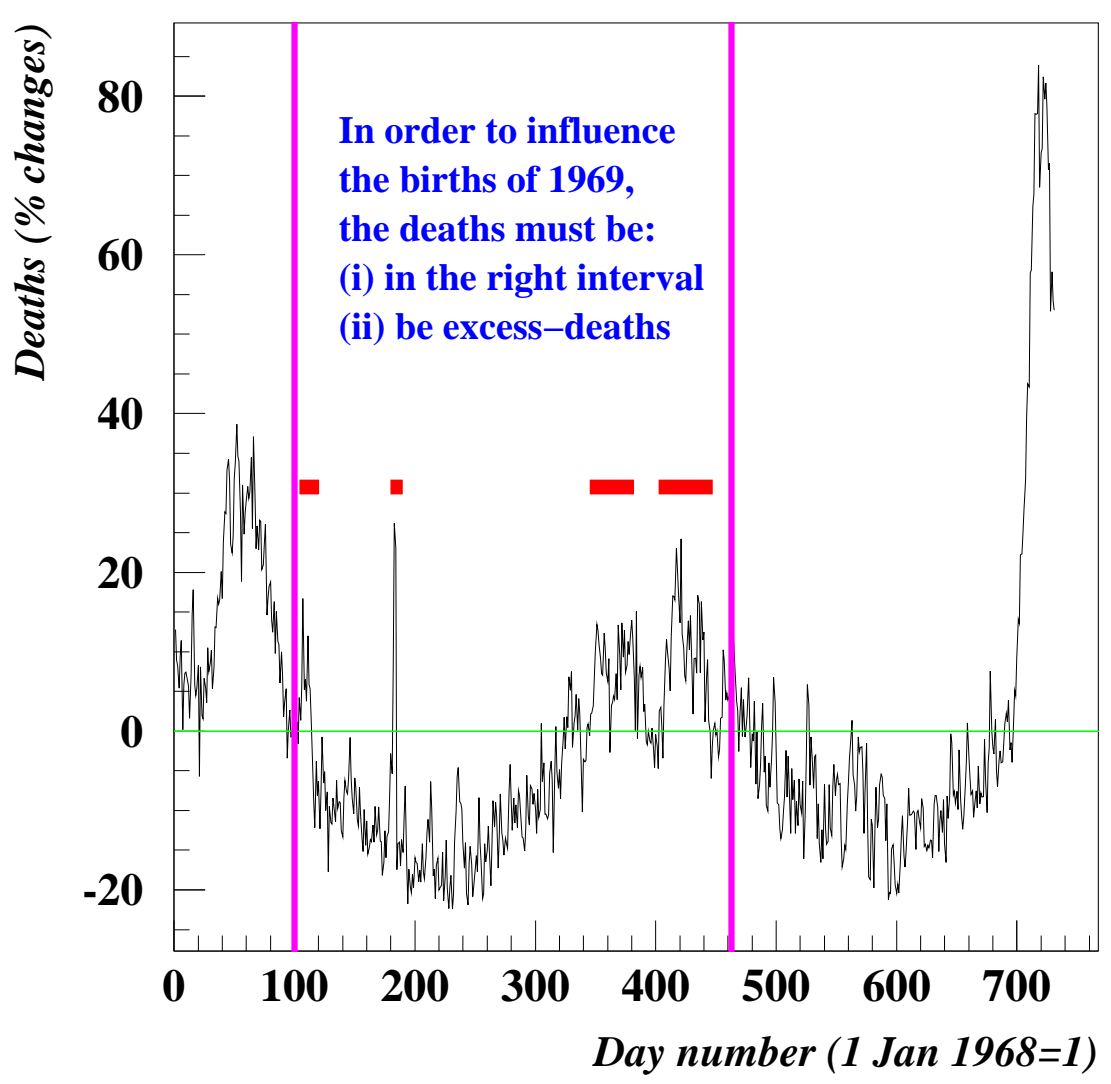

Fig. 5 Removal of the death-nobirth effect, for daily births, France 1968-1969. This figure explains why in 1969 the removal of the death-nobirth effect has only a small incidence. Sources: Same website as for Fig. 4.

In order to affect the births of 1969 , the deaths must occur in the time interval shown in magenta in Fig.5.3; within the two years 1968-1969 this defines a specific time interval. It turns out that in this interval deaths are mostly below average; there are only small sub-intervals (marked in red) which have excess-deaths. Therefore the curve of the births during 1969 is only slightly changed. The fact that despite this small impact the standard deviation of births is nevertheless reduced by $11 \%$ shows that the correction works indeed in the expected way that is to say by pushing up the

${ }^{3}$ More precisely we have taken a pregnancy duration of 267 days as explained in Richmond et al. (2018a). 
troughs; the modified birth curve is omitted because visually the changes are rather subdued.

\section{Removal of the death-nobirth effect for monthly births}

Graphically the effect of the removal of the death-nobirth effect will become clearer at the level of monthly births. This is shown in Fig. 6.

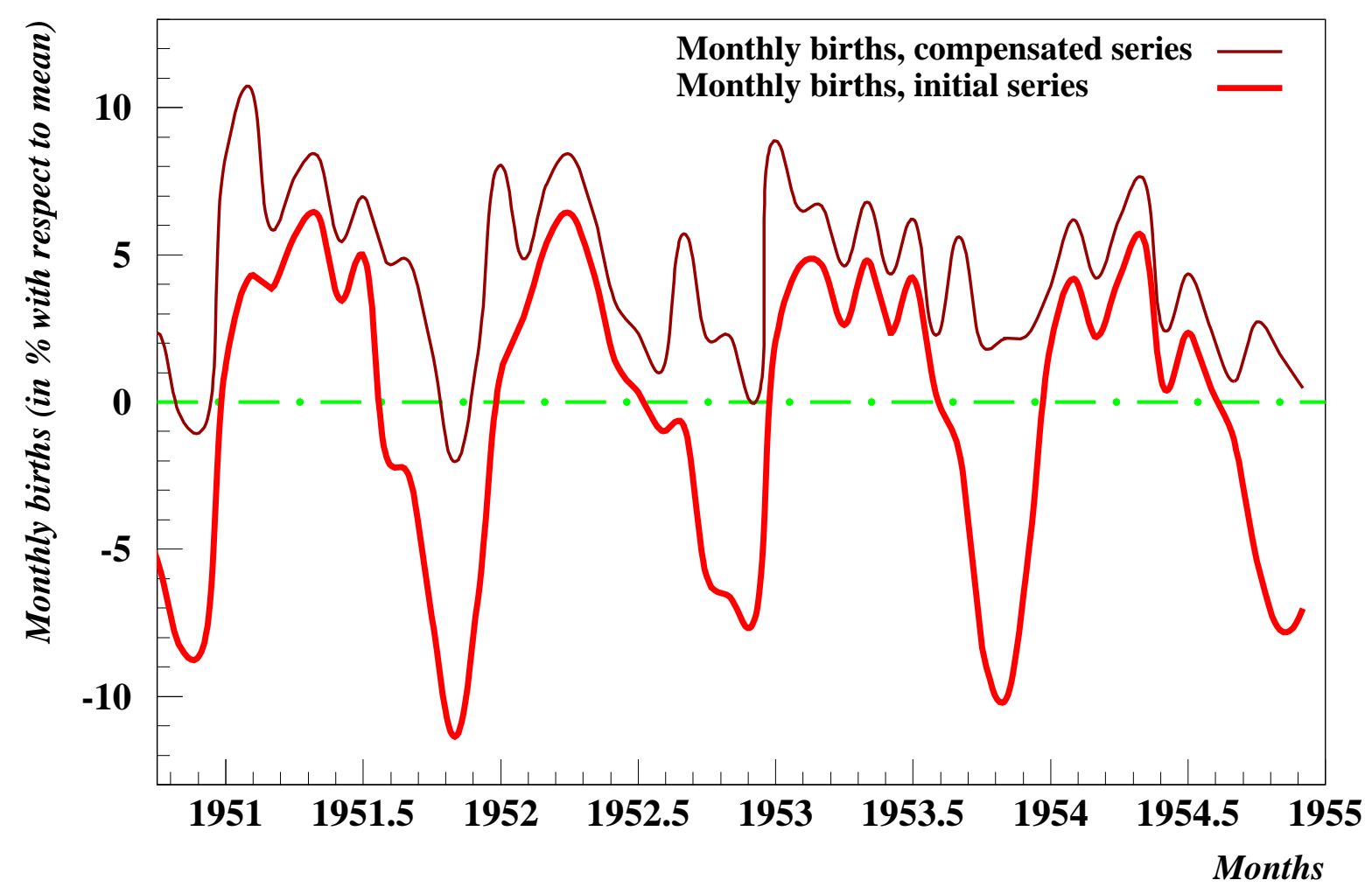

Fig. 6 Removal of the death-nobirth effect, France 1950-1954. For the compensation we used the regression coefficients $a, b$ obtained in Fig. $4 \mathrm{~b}$; then the compensation was done as follows: $\Delta b=a \Delta d+b$, where $\Delta d, \Delta b$ are the excess deaths and excess births respectively; then $b_{c}=b_{i}+\Delta b$ where $b_{i}, b_{c}$ are the initial and compensated birth series respectively. Note that $\Delta b$ is negative and that this compensation was done only when there were excess deaths, that is to say when the death series was below its average. In the graph, the compensated series was translated somewhat upward to avoid a superposition with the initial series in all the intervals in which there are no excess-deaths and therefore no compensation. Sources: Same website as for Fig. 4.

It can be seen that all deep troughs occurring around September are replaced by fairly shallow troughs. This corresponds to a reduction of the standard deviation from 5.11 for the initial series to 2.90 for the compensated series.

The success of this procedure raises the question of whether nevertheless there remain some deep troughs outside of the time interval shown in Fig. 5. This point is discussed below; it will be seen that in recent decades a new phenomenon appeared which brought about unexpected birth troughs 
Before considering a decade which shows birth troughs that do not match expectations, let us show one in which the death-nobirth effect accounts for the observed birth troughs (Fig. 7a).
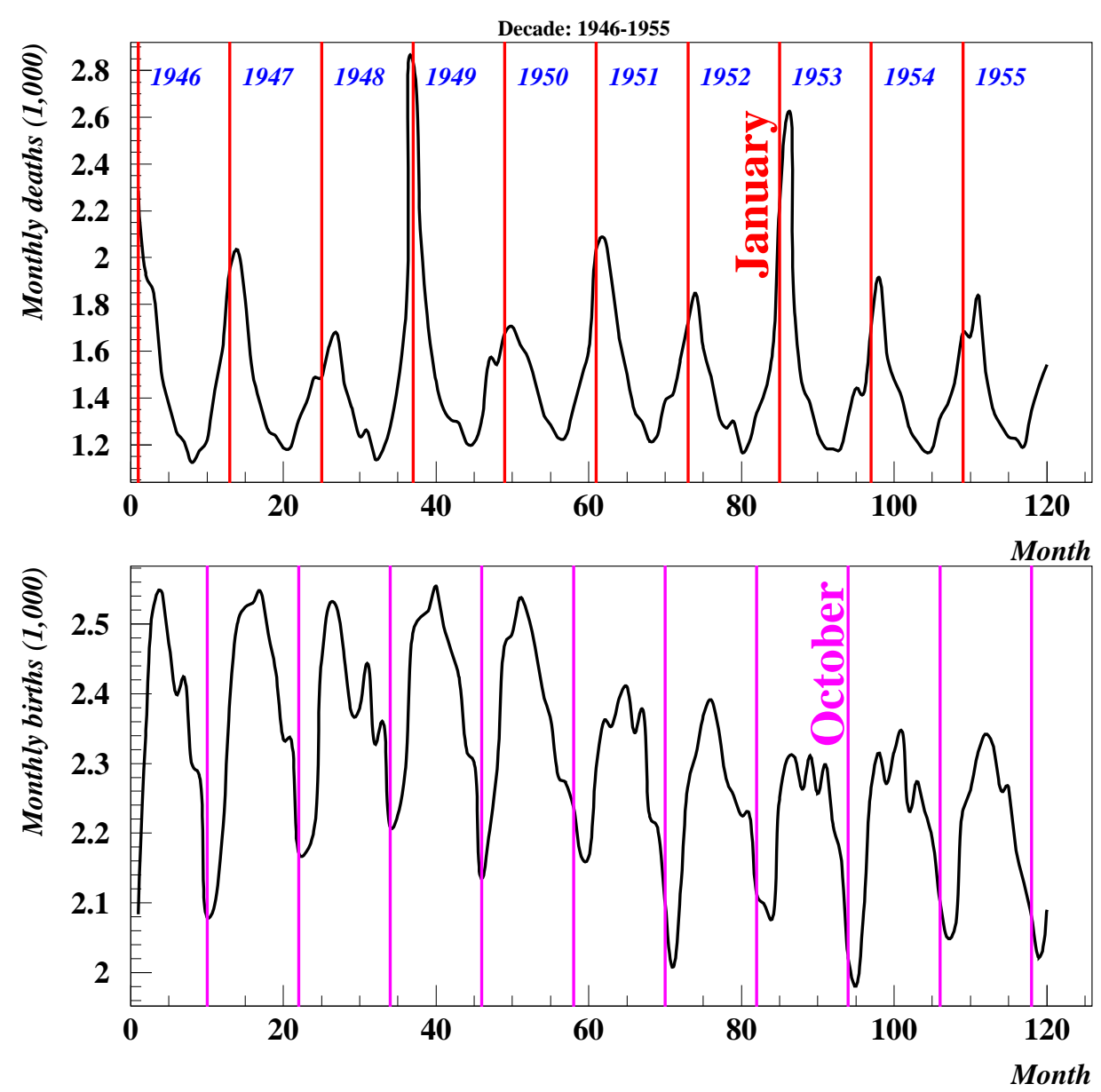

Fig. 7a Illustration of the death-nobirth effect. The data are for France and correspond to monthly deaths and births divided by the number of days of each month. All red lines of the death graph indicate January and all magenta lines of the birth graph indicate October (i.e. January+9months). It can be seen that the birth troughs follow the death spikes with the expected time lag of 9 months. However, as shown in the next graph, in the decades after 1990 the birth troughs moved progressively to the left of October. Source: Same website as for Fig. 4.

In Fig.7b we have birth troughs which follow a different pattern. Actually it is a fairly loose pattern: in 1997 the birth trough occurs in March, in 1998 there is no clearly defined trough, in 1999, 2000, 2001 the troughs occur in January, in 2002,2003 it is in February, in 2004 there is a double dip in February and May one of which may be due to the death spike of August 2003. In short, instead of the expected troughs we see others that were unexpected.

What explanations can one give?

- More than the apparition of a new effect it is the apparent disappearance of the death-nobirth effect which is surprising. In fact, it does not really disappear. The 


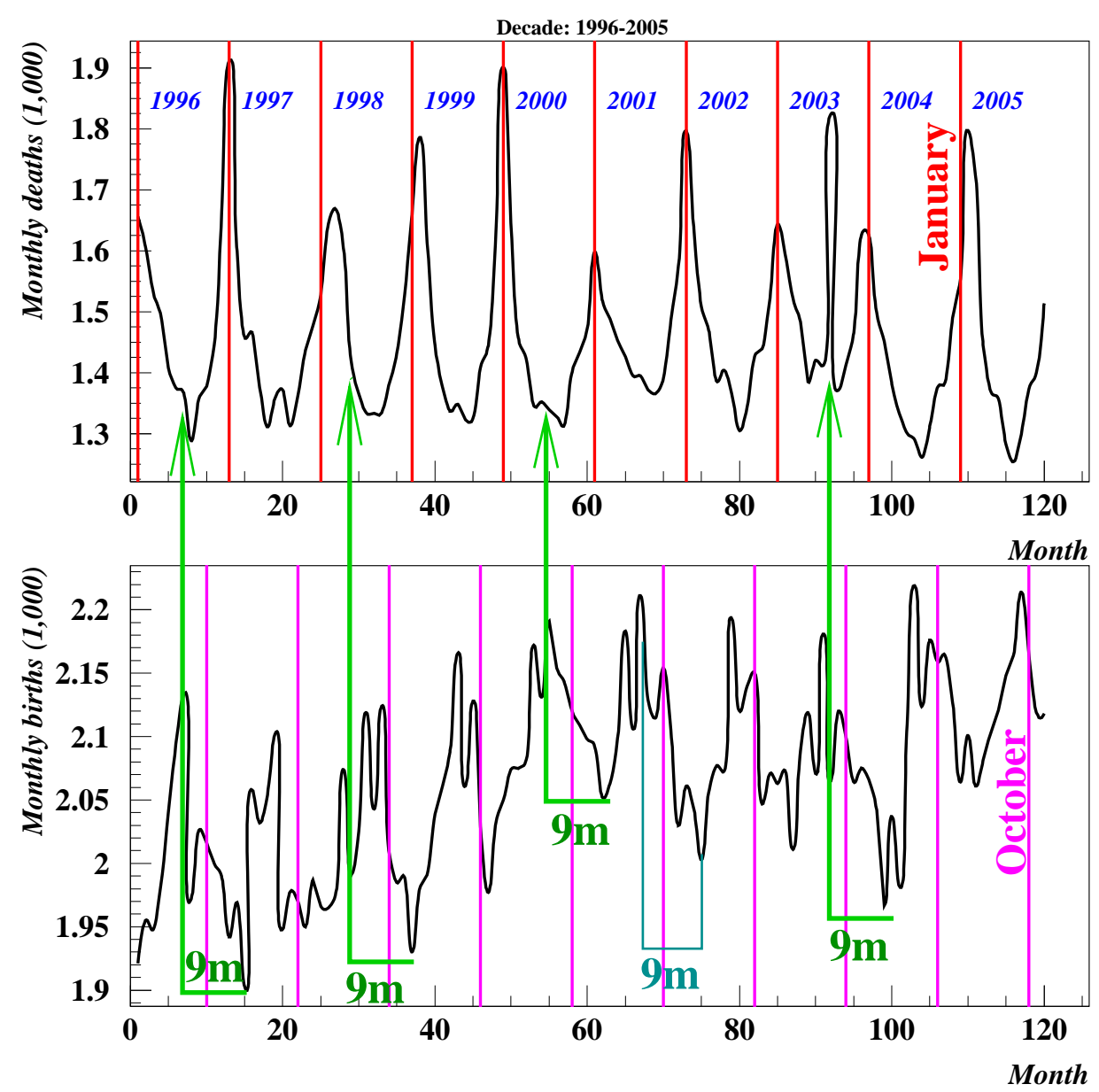

Fig. 7b Apparition of a new effect which produces birth troughs not related to death spikes. Here, in most of the cases the time lags between death spikes and birth troughs are longer than 9 months. However the death spike associated with the heat wave of August 2003 is followed by a birth trough 9 months later (i.e. in May 2004). In addition, we observe a time lag close to 9 months between birth peaks and birth troughs (see the U shaped line in cyan) for which an explanation is suggested in the text. Source: Same website as for Fig. 4.

October dips are still present but in an hidden way due to the presence of lower dips.

- What can be the origin of the new troughs?

In comparison with Fig.7a the births dips appear more disorderly, often for instance displaying a double dip. Similarly the birth peaks appear also more disorderly with double dips often being preceded by double spikes. This observation leads us to a possible explanation which is based on the fact that for a couple conception at time $t$ excludes conception at a later time at least until after birth. If all couples in age of having a baby would conceive one in October, then during the 9 subsequent months there would be no conceptions. As a result, one would see a huge birth peak in October +9 months=July followed by a 9-month interval without any birth. In the real world where couples are not highly synchronized one may expect a conception peak to be followed by a conception trough 9 months later. This is indeed what we observe (see the $\mathrm{U}$ shaped line in cyan color). For the sake of brevity this effect will 
be referred to as the single conception effect.

Actually, it is quite possible that this effect contributed in some way to the deathnobirth effect for in Fig.7a one sees that the time interval between birth peaks and birth dips is comprised between 6 and 9 months, that is to say not too different from 9 months.

A last question has to be answered, namely why did the single conception effect suddenly overcome the death-nobirth effect? A possible answer may be found in the width of the birth peaks. Comparison of Fig. $7 \mathrm{a}$ and $7 \mathrm{~b}$ shows that they become much more narrow which implies more synchronized conceptions, therefore followed by deeper conception dips.

\section{Influence of the religious environment on conceptions}

There are religious precepts that rule sexual intercourse during special periods. For instance, during the month of Ramadan, sexual activity is not allowed during the day but is permitted during the night. The orthodox religion advises against sexual activity during Lent which in Orthodox countries is a 7-week period (49 days) preceding Easter. In present day Catholic and protestant religions there are no specific precepts on this point. However, although the scriptures do not say anything in this respect in past centuries the Catholic Church prohibited sexual activity not only during Lent (40 days before Easter) but also in many other periods, e.g. 40 days before Christmas, 40 days after giving birth, or on all the nights from Saturday to Sunday.

Conversely, it can be mentioned that on some specific days, sexual intercourse is encouraged by religious rules. That is the case of Saturday (or more precisely Shabbat which starts already on Friday night) in the Jewish religion.

In connection with the present paper, we will address two questions.

(1) Is there empirical evidence showing that some religious precepts affected the pattern of births?

(2) Is it possible to document the impact of religious rules in the course of time and across countries?

\section{Empirical evidence: the case of the Fire Horse year in Japan}

Probably the easiest way to show the impact of beliefs on births can be found in Japan. The reason which makes it easy is the fact that one does not need to use monthly or even daily data. Annual birth data are sufficient. The population pyramid of Japan based on the census of 2000 presents a mysterious discontinuity for people aged 34 that is to say who are born in 1966. The number of people (both males and females) born in this year is much smaller than in 1965 or in 1967. The difference is of the order of 30\%. Further investigation reveals that 1966 was a Hinoeuma year, 
which means a Fire Horse year in the Chinese calendar 4 . Girls born in that year grow up to be known as "Fire Horse women" and are reputed to be headstrong and to bring bad luck to their families and to their husbands. In 1966, as a baby's sex could not be reliably identified before birth, there was a sharp fall in birth rate. This fall was due partly to a fall in conceptions and partly to an increase in abortions. According to the Chinese calendar, Fire Horse years occur every 60 years; thus, the three previous one were in 1906, 1846 and 1786. It is a natural question to see if the effects in those years were similar to the one in 1966. For 1786 there are no data but for the three subsequent years the answers are given in Table 3.

Table 3: Birth reductions in the last three Fire Horse years

\begin{tabular}{lccc} 
& 1846 & 1906 & 1966 \\
\hline Fall in annual births (\%) & $-11 \%$ & $-11 \%$ & $-24 \%$ \\
\hline
\end{tabular}

Notes: The percentages refer to the differences between the year under consideration and the mean of the 9 other years in the same decade. The high reduction in 1966 is due to two factors. (i) Contrary to what happened in 1846 and 1906, in 1966 the male-female sex ratio at birth increased only by $1.3 \%$ instead of $20 \%$ in 1846 and $4.3 \%$ in 1906; this means almost no infanticide in 1966. (ii) In 1946 there was a sharp fall in births which generated a small cohort; then, in 1966 this cohort reached the age of 20 at which point it started to have children.

Source: Roehner (2007, p. 42).

\section{Empirical evidence: the case of Lent in Romania}

Why should one focus on the case of Lent in Romania? There are two good reasons for that.

- As mentioned above, in contrast with the Catholic or Protestant religions, the Orthodox religion has clearly defined rules regarding sexual relations during Lent. In the census of $2011,86 \%$ of the Romanian population identified as part of the Eastern Orthodox Church.

- Because the time of Lent changes from year to year it is a much better "marker" than a fixed religious day. The reason is obvious. A single observation may display a reduction in births but it will not tell us the reason of this reduction. On the contrary, if on successive years the birth reductions follow closely the Lent time-intervals, then there can be little doubt that Lent is the causal factor.

The effect of religious rules on conception during Lent was investigated in Herteliu et al. (2015). For such a study one of the main difficulties is the fact that strictly speaking monthly birth data are not detailed enough to follow the shifts of Lent. One needs weekly or daily data. Fortunately, the Romanian Bureau of the Census had the

\footnotetext{
${ }^{4}$ Because the Chinese New Year occurs in late January, the Fire Horse Year does not exactly coincide with 1966; in fact, it started on 21 January 1966 and ended on 8 February 1967.
} 
clever idea to ask their birthday dates to all persons surveyed in the censuses of 1992 and 2002.

Naturally, the oldest persons may not remember their birthday with absolute accuracy. In order to limit this uncertainty the data set was limited to the persons born after 1905; thus, the persons included in the data set were less than 87 year old in 1992. Because the size of a cohort decreases rapidly after 65 the dataset would include only small samples of persons born between 1905 and 1927.

For the whole period from 1930 to 2000 the reduction of conceptions during Lent was found to be on average equal to: $14.1 \% \pm 1 \%$.

In contrast there was almost no reduction during the 40-day Nativity Fast (also called "Advent") that precedes Christmas.

\section{Comparative perspective across countries}

On account of what we said above, one would expect a smaller reduction effect in Catholic or Protestant countries than in Orthodox countries How can one test this conjecture?

As daily or weekly birth data will not be available in the early time periods that we wish to consider we made the following simplified argument which will allow us to use monthly data.

(1) Firstly, the comparison will be made on a time interval before World War II, for instance 1931-1935 because this will allow us to use the monthly data given in Bunle (1954).

(2) Secondly, we observe that in western countries Easter is on average on 8 April. Indeed, averages performed over several 30-year intervals always lead to the same date. Note that this is for Catholic and Protestant countries. For Orthodox Easter the average date is somewhat later, namely on 21 April. As in western countries Lent lasts 40 days, on average western Lent will last from 1 March to 8 April which means that if we identify Lent with March we will miss only $8 / 40=20 \%$.

For Orthodox Easter the situation is less favorable. As Lent lasts 49 days it will be from 1 March to 21 April and will therefore be divided more equally (4/7 against 3/7) between March and April. By identifying Lent with March we will miss 21/49= $43 \%$.

There is an additional factor which makes the approximation more acceptable. If the religious injunction is followed fairly closely one would expect a rebound of conceptions after Easter. Such a rebound can indeed be observed; for instance in Bulgaria conceptions surged from 672 in March to 983 (+46\%) in April. In western countries a surge can also be observed but which is much smaller. For instance in France from March to April conceptions rise from 939 to $1042(+11 \%)$. 
In other words, in Orthodox countries any reduction between 1 and 21 April might be reduced by a surge of conceptions in the rest of the month. Thus, even in Orthodox countries, March appears to be a more appropriate estimate of Lent than April.

If one accepts the previous argument the conception reduction due to Lent can be represented by the ratio5:

$R=$ conceptions of March divided by one half of the total conceptions of February and April By adding 9 months this ratio will get translated into an estimate for births.

Table 4: Conception reduction during Lent, 1931-1935

\begin{tabular}{llcccc}
\hline \hline Conceptions in: & Feb. $(f)$ & Mar. $(m)$ & Apr. $(a)$ & $R=m /[(f+a) / 2]$ \\
\cline { 2 - 3 } & 891 & 688 & 983 & 0.73 \\
Romania & 911 & 686 & 919 & 0.75 \\
& & & & 0.95 \\
France & 985 & 930 & 1042 & 0.94 \\
Spain & 911 & 947 & 1096 & 0.97 \\
& & & & 1.00 \\
Norway & 857 & 899 & 992 & 979 \\
Sweden & 904 & 941 & 979 \\
\hline
\end{tabular}

Notes: $R$ equal to 1 would mean that March which represents Lent has same conceptions as the two adjacent months that is to say no reduction due to Lent. The monthly numbers given in the table are an index which sums up to 1,000 for one year. Bulgaria and Romania are Orthodox countries, France and Spain are Catholic countries, Norway and Sweden are Protestant countries.

Source: Bunle (1954, p. 92-93).

\section{Comparative perspective across time}

One would expect a decrease of the reduction effect in the course of time. How can one test this conjecture?.

The data given in Herteliu et al. (2015) allow us to follow the reduction from about 1930 to 2000. Needless to say, one would not be surprised to see a fairly sharp fall of the impact of religion during the 40 years of Communist regime. However, in order to better assess the rapidity of the fall we need to compare it with a non-Communist country. If we use again the approximation which allows us on average to identify Lent with March, we can use French monthly birth data which cover a broad time interval ranging from 1650 to 2000. The comparison presented in Fig. 8 shows that in Romania the fall is about 6 times faster than it was in France.

\section{Conclusion}

In this paper we have given interpretations of several kinds of birth rate troughs.

\footnotetext{
${ }^{5}$ Because of the "surge effect" the conceptions of April will be higher than they would be without such an effect. As a result, $R$ will be lower than it should be.
} 


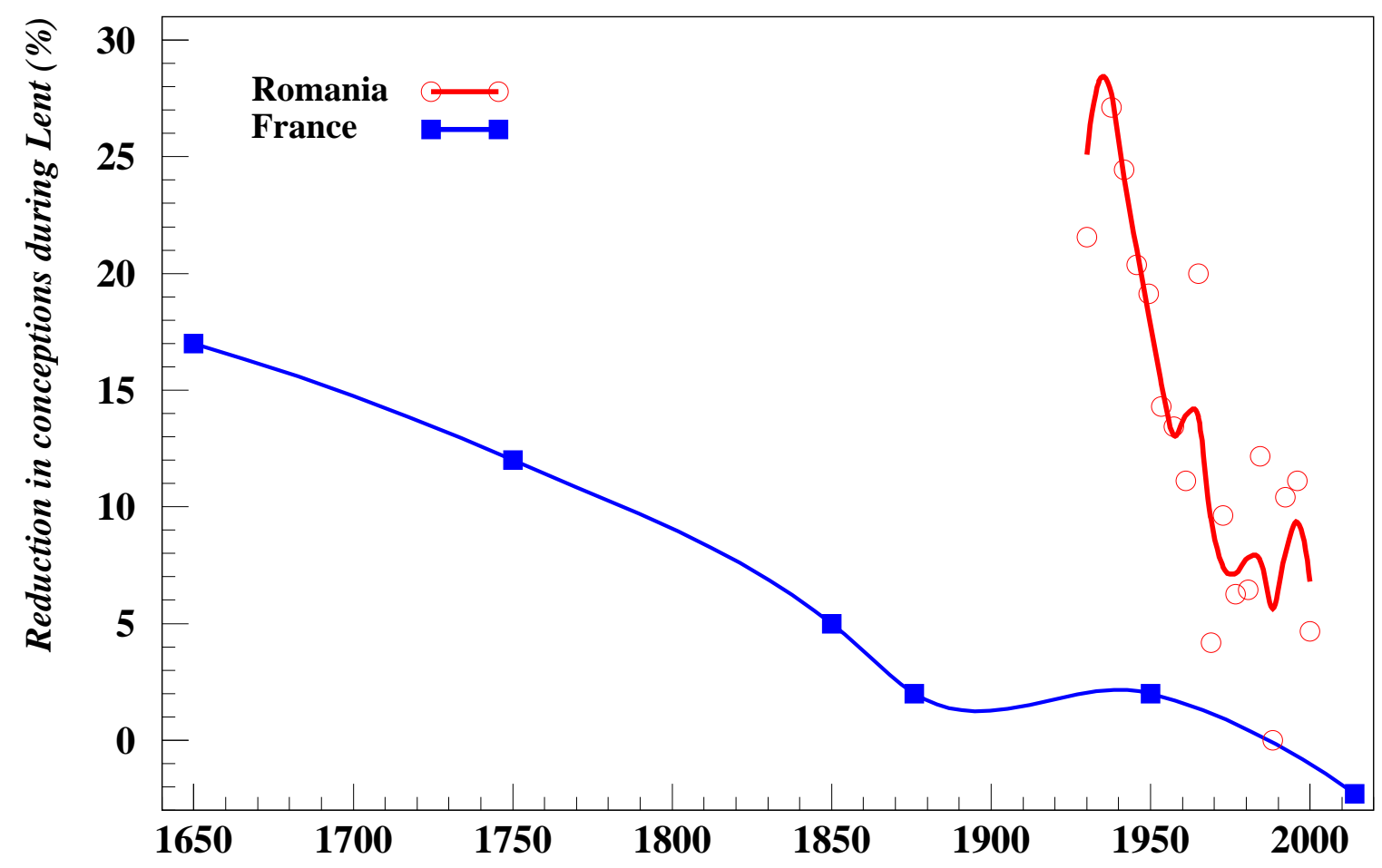

Fig. 8 Reduction of conceptions during Lent in France and Romania. The results for France are based on monthly birth data whereas those for Romania are based on daily data which is why they have a larger dispersion. Actually, prior to the 19th century, the records give the dates of baptisms. In order to accept them as proxies of birth dates one must assume that baptism followed birth fairly closely. Overall the fall of the Romanian curve is 6 times steeper than the fall of the French curve. Sources: Herteliu et al. (2015), Houdaile (1979, 1985), Régnier-Loilier 2010b.

However, the origin of birth rate peaks remains an open question 6 .

The main hallmark of the rules that we have studied is that, to varying degrees, they are valid in all countries for which we were able to find reliable data. For instance, although in present-day France the influence of religious beliefs is so small that it can hardly be measured, the analysis of historical data shows that in the past this effect did indeed exist.

Obviously, this provides an objective way to test and assess the beliefs of people. We plan to extend this investigation further as soon as new data become available. For instance, when monthly birth data become available for China it would be interesting to test if there are conception surges in the "Golden week" (1-7 October) or during the "Spring festival", that is to say the week centered on the Chinese New Year.

\footnotetext{
${ }^{6}$ There is a birth rate peak in late September (see Fig. 1,2,3 ) which corresponds to a conception peak in December. It may be due to the Christmas celebration. However, how can one prove that it is really due to Christmas rather than to some other factor?
} 


\section{Appendix A: A historical view of previous studies}

Monthly birth data series became available in European countries in the early 19th century and in the United States about one century later (due to the fact that it took some time to set up registration procedures in all states). Explaining the monthly distribution of births is a question which attracted the attention of researchers very early. One of the first studies was published in 1831 by Louis René Villermé (1782-1863), a French physician and a pioneer of social epidemiology. In several respects his article is typical of those published at that time and it may be of interest to understand in what aspects it differs from present-day publications.

(1) It is a long article of one hundred pages.

(2) It is a comparative study which uses data from many countries: France of course, but also Great Britain, Germany (or rather Bavaria, Prussia, Wurtemberg for at that time Germany was not unified), Italy, the Netherlands, Russia, Sweden. It uses also data from various time periods going back for some places (e.g. Paris) to the 17th century. This kind of comparative methodology remained in use during the whole 19th century. Recall that Durkheim's renowned study of suicide (1897) used exactly the same methodology which, needless to say, was borrowed from physics where experimenters also perform as many observations as possible in order to understand any new phenomenon..

(3) Interestingly, 30 pages of the paper are statistical tables which contain all the data used by the author. Several of these data sets are still useful nowadays, as for instance the monthly birth numbers for Belgium and the Netherlands from 1815 to 1826. The thanks expressed in the notes of the tables reveal that the author had personal contacts with several foreign scholars from whom he received many of his data.

(4) In line with other scholars of the 19th century (e.g. Alfred Espinas, 1878) Villermé does not hesitate to includes in his study observations about animal species. This is of course in accordance with the perspective of comparative analysis. He observes for instance that for foxes, hares and wolves conception takes place between December and February which is fairly different from humans for whom the conception peak is in spring.

(5) Basically the paper states most of the general rules we currently know about. In other words, progress seems to have been fairly limited.

Let us now mention some more recent studies, e.g. MacFarlane (1974), Seiver (1985), Lam et al. (1994). Huber et al. (2011). They are of two types. The first three are fairly broad studies whereas the last one focuses on a very specific point. For all of them one could say that they provide evidence but no real understanding; 
actually not even an "observational understanding,'7This is fairly obvious for the specialized paper because in this case the question it addresses is too specific to be understood from any basic principles that may exist. The same is true for the broad papers yet for a different reason. Although they offer evidence, even in some cases such as MacFarlane (1974) comparative evidence for several countries, they refrain from raising any questions. For instance, Lam et al. (1994) show that between the monthlu birth patterns of Britain and the US state of Georgia there is a 6 month time lag. This difference comes of course as a surprise for two regions that are fairly similar in many respects. However the authors do not try to explain it. Even if the reason cannot be identified, would it not be useful to point out that, at least for now, there is no satisfactory explanation? This might stimulate more sharply targeted research.

\section{References}

Bunle (H.) 1954: Le mouvement naturel de la population dans le monde de 1906 à 1936. [Vital statistics of many countries worldwide from 1906 to 1936.] Editions de l'Institut National d'Etudes Démographiques, Paris.

Espinas (A.) 1878, 1935: Des sociétés animales [on animal societies]. Thesis of the university of Paris. Republished in 1935, Félix Alcan, Paris.

[The title looks more original when one remembers that the author was in fact a sociologist.]

Herteliu (C.), Ileanu (B.V.), Ausloos (M.), Rotundo (G.) 2015: Effect of religious rules on time of conception in Romania from 1905 to 2001. Human Reproduction 30,9,2202-2214.

Houdaille (J.) 1979: Mouvement saisonnier des conceptions en France de 1740 à 1829 [Seasonal pattern of conceptions in France from 1740 to 1829.] Population 2,452-457.

Houdaille (J.) 1985: Le mouvement saisonnier des naissances dans la France rurale de 1640 à 1669. [Seasonal pattern of conceptions in rural France from 1640 to 1669.] Population 2,360-362.

Huber (S.), Fieder (M.) 2011: Perinatal winter conditions after later reproductive performance in Romanian women: intra and intergenerational effects. American Journal of Human Biology 23,546-552.

Lam (D.A.), Miron (J.A.) 1994: Global patterns of seasonal variation in human fertility. Annals of the New York Academy of Sciences 709,9-28.

Lerchl (A.) 2005: Where are the Sunday babies? Observation on a marked decline on weekend births in Germany. Naturwissenschaften 92,592-594.

Lerchl (A.), Reinhard (S.C.) 2008: Where are the Sunday babies? II. Declining

\footnotetext{
${ }^{7}$ By this expression we mean a rule valid in all countries where data are available which gives it real predictive power.
} 
weekend birth rates in Switzerland. Naturwissenschaften 95,161-164.

Lerchl (A.) 2008: Where are the Sunday babies? III. Caesarean sections, decreased weekend births, and midwife involvement in Germany. Naturwissenschaften 95,165-170.

MacFarlane (W.V.) 1974: Seasonal cycles of human conception. Progress in biometeorology A, Vol.1,557-577 and 711-713.

Régnier-Loilier (A.) 2010a: Évolution de la saisonnalité des naissances en France de 1975 à nos jours [Changes in the seasonal birth pattern in France from 1975 to 2006]. Population, 65,1,147-189.

Régnier-Loilier (A.) 2010b: Évolution de la répartition des naissances dans l'année en France [Changes in the seasonal birth pattern in France]. Actes du XVe colloque national de démographie [Proceedings of the 15th national conference on demography, 24-26 May 2010] Published by the "Conférence Universitaire de démographie et d'étude des populations".

Rey (G.), Fouillet (A.), Jougla (E.), Hémon (D.) 2007: Vagues de chaleur, fluctuations ordinaires des températures et mortalité en France depuis 1971 [Heat waves, temperature fluctuations and mortality in France from 1971 to 2003]. Population 62,3,533-564.

Richmond (P.), Roehner (B.M.) 2018a: Coupling between death spikes and birth troughs. Part 1: Evidence. [Available on the arXiv website: arXiv: 1801.04533.]

Richmond (P.), Roehner (B.M.) 2018b: Coupling between death spikes and birth troughs. Part 2: Comparative analysis of salient features. [Available on the arXiv website: arXiv: 1801.04535.]

Sardon (J.-P.) 2005: Influence des épidémies de grippe sur la fécondité. [Influence of influenza epidemics on fertility.] In: Bergouignan (C.) et al.: "La population de la France. Evolutions démographiques depuis 1946, Vol. 1, p. 413-417. [This short but interesting paper is focused on the effect of the epidemic of 1957 on births in European countries.]

Seiver (D.A.) 1985: Trend and variation in the seasonality of US fertility, 1947-1976. Demography 22,1,89-100.

Vicedo-Cabrera (A.), Ragettli (M.), Schindler (C.), Röösli (M.) 2016: Excess mortality during the warm summer of 2015 in Switzerland. Swiss Medical Weekly, 5 December 2016.

Villermé (L.R.) 1931: De la distribution par mois des conceptions et des naissances de l'homme [On the monthly distribution of human conceptions and births]. Annales d'Hygiène Publique et de Médecine Légale, Série 1, No 5,55-155. 